\title{
A meta-analysis and The Cancer Genome Atlas data of prostate cancer risk and prognosis using epithelial cell adhesion molecule (EpCAM) expression
}

\author{
Yu Hu${ }^{1 \dagger}$, Qiong $\mathrm{Wu}^{1+}$, Jialin Gao ${ }^{2}$, Yongrui Zhang ${ }^{2}$ and Yuantao Wang ${ }^{2^{*}}$
}

\begin{abstract}
Background: Epithelial cell adhesion molecule (EpCAM) expression has been reported in many types of cancer, including prostate cancer (PCa). However, the role of EpCAM expression remains inconsistent. We conducted a meta-analysis to assess the clinicopathological and prognostic significance of EpCAM expression in PCa.
\end{abstract}

Methods: Publications were searched online using electronic databases. The available data were obtained from The Cancer Genome Atlas (TCGA). The odds ratios (ORs) or hazard ratios (HRs) with their 95\% confidence intervals (Cls) were calculated.

Results: We identified seven studies in which immunohistochemistry was used and that included 871 prostatic tissue samples. EpCAM expression was significantly higher in PCa samples than in benign and normal tissue samples $(\mathrm{OR}=77.93, P=0.002 ; \mathrm{OR}=161.61, P<0.001$; respectively). No correlation of EpCAM overexpression with PT stage and lymph node metastasis was observed; however, EpCAM overexpression showed a significant correlation with Gleason score $(\mathrm{OR}=0.48, P=0.012)$ and bone metastasis $(\mathrm{OR}=145.80, P<0.001)$. Furthermore, TCGA data showed that EpCAM overexpression was not closely correlated with age, pT stage, lymph node metastasis, number of lymph node, prostate-specific antigen level, Gleason score, biochemical recurrence, and overall survival. Based on multivariate Cox proportional-hazards regression analysis, a significant correlation was observed between EpCAM overexpression and 5-year worse biochemical recurrence free-survival.

Conclusions: EpCAM overexpression may be correlated with the development of bone metastasis and worse biochemical recurrence free-survival of PCa. Further studies are needed to verify these findings.

Keywords: EpCAM, Expression, Bone metastasis, Prognosis, Prostate cancer

\section{Background}

Prostate cancer $(\mathrm{PCa})$ is the second commonest malignancy and the fifth leading cause of cancer-related deaths in men [1]. Based on GLOBOCAN estimates, approximately 1.3 million new cases were clinically diagnosed with PCa in 2018, leading to approximately 359, 000 PCa-related deaths worldwide [1]. Several therapeutic strategies, including radical prostatectomy and

\footnotetext{
* Correspondence: wangyuantaobs@163.com

${ }^{\dagger}$ Yu Hu and Qiong Wu contributed equally to this work.

${ }^{2}$ Department of Urology, The First Hospital of Jilin University, No. 71 Xinmin

Street, Changchun 130021, Jilin, China

Full list of author information is available at the end of the article
}

radiotherapy, have shown a better clinical outcome for patients with early-stage $\mathrm{PCa}[2,3]$. In contrast, patients with advanced stage $\mathrm{PCa}$ have distant metastases and consequently, worse prognosis because of the lack of the effective treatment options $[4,5]$. Therefore, a novel molecular biomarker is required to improve the prognosis of patients with $\mathrm{PCa}$.

Cancer stem cells (CSCs) are a small group of cells within tumors and are responsible for self-renewal, uncontrolled differentiation, and tumorigenicity $[6,7]$. CSCs contribute to cancer development, progression, and metastasis [8-10]. Epithelial cell adhesion molecule (EpCAM), known as epithelial-specific antigen (ESA) or

(c) The Author(s). 2019 Open Access This article is distributed under the terms of the Creative Commons Attribution 4.0 International License (http://creativecommons.org/licenses/by/4.0/), which permits unrestricted use, distribution, and 
CD326, a membrane glycoprotein, plays an important role in $\mathrm{Ca} 2+$ independent hemophilic cell-to-cell adhesion, cell signaling, migration, proliferation, and differentiation [11, 12]. The presence of CSCs in PCa may partially play a role in cancer progression, metastasis, and chemoresistance $[13,14]$. EpCAM is identified as a CSC marker and a potential therapeutic target for cancer [15]. EpCAM is expressed in many types of human cancer, such as breast cancer, gastric cancer, and colorectal cancer [16-18]. Recent studies also demonstrated that high EpCAM expression may predict poor clinical outcome in breast cancer [19], ovarian carcinoma [20], and hepatocellular carcinoma [21]. Some studies reported that EpCAM was frequently expressed and associated with worse prognosis of patients with PCa [22, 23].

The development of $\mathrm{PCa}$ develops involves the transition of normal epithelium to benign prostatic epithelium, and subsequent progression to malignant carcinoma through multiple sequences [24-26]. The role of EpCAM expression in PCa development and progression remains controversial. Ni 2013 et al. reported that the frequency of EpCAM expression was similar in $\mathrm{PCa}$ and benign prostatic tissue samples [22]. In contrast, Li et al. showed that EpCAM expression was notably higher in PCa than benign prostatic tissue samples [27]. Thus, the primary objective of this study was to identify the role of EpCAM in determining the risk of PCa development. The secondary objective was to perform a meta-analysis to assess the clinicopathological and prognostic value of EpCAM in PCa.

\section{Methods}

\section{Search strategy from meta-analysis}

This meta-analysis was conducted based on the preferred reporting items for systematic reviews and metaanalyses (PRISMA) statement criteria [28]. Articles published until December 1, 2018 were systematically searched in PubMed, EMBASE, Cochrane Library, EBSCO, Wanfang, and CNKI databases. The following keywords and search terms were used: "epithelial cell adhesion molecule OR EpCAM OR Ep-CAM OR CD326 OR 17-1A antigen OR GA733 OR CO17-1A OR EGP OR KS1-4 OR ESA OR MOC31 OR BerEP4 OR TACSTD1 OR TROP1," "prostate OR prostatic," "cancer OR carcinoma OR tumor OR neoplasm." We also manually screened the reference lists of the eligible studies to find other potential publications.

\section{Selection criteria from meta-analysis}

Studies that met the following criteria were selected: 1) patients were diagnosed with $\mathrm{PCa}$ by histopathological examination; 2) the immunohistochemical assessment of EpCAM using anti-EpCAM antibody was defined as a positive based on the publications; 3 ) studies provided the available data for the association of EpCAM expression between $\mathrm{PCa}$ and nonmalignant control groups; 3) studies provided the available data for the correlation of EpCAM expression with the clinicopathological features of patients with $\mathrm{PCa}$; 4) studies provided sufficient data to evaluate the prognostic role of EpCAM expression using multivariate Cox proportional-hazards regression analysis. Only the latest or more accurate study was included when authors published several papers using duplicated sample data. The exclusion criteria were as follows: 1) letters, case reports, editorials, and studies conducted in animals and cell lines; 2) studies that lacked sufficient data.

\section{Data extraction from meta-analysis}

Data were extracted from all eligible studies independently by two authors. Discrepancies were resolved by a third author. The following information was extracted: name of the first author's names, year of publication, country, ethnicity and age of the patients, tumor stage, detection method, antibody, cut-off values, expression frequency, number of cancer patients and nonmalignant controls, clinicopathological characteristics, and survival data.

\section{TCGA dataset}

The RNA-sequencing PCa data and corresponding clinical information were downloaded from TCGA (https:// cancergenome.nih.gov/). Finally, 495 PCa patients with the available clinical information and 52 normal prostatic samples were included.

\section{Statistical analysis}

Data were obtained from the original articles. The corresponding author of each study with an available email was also contacted to request useful information on missing or incomplete data. The pooled odds ratios (ORs) and the corresponding 95\% confidence intervals (CIs) were used to estimate the difference in EpCAM expression between $\mathrm{PCa}$ and nonmalignant controls. The association of EpCAM expression with the clinicopathological parameters of $\mathrm{PCa}$ were also calculated using pooled ORs with 95\% CIs. Based on multivariate Cox proportional-hazards regression analysis, the overall hazard ratios (HRs) with 95\% CIs were used to determine the prognostic impact of EpCAM expression. The statistical heterogeneity between studies was estimated using the Cochran's Q statistic [29]. The random-effects model (DerSimonian and Laird method) was used to improve the reliability of the data [30]. When significant heterogeneity was present $(P<0.1)$, a sensitivity analysis was performed to evaluate the influence and stability of one study on the results by deleting a single study [31]. Publication bias was measured using Egger's linear regression test ( $\geq 10$ studies) [32]. Meta-analysis was 
performed using Stata software (version 12.0, Stata Corporation, College Station, TX, US).

For TCGA dataset, the difference in EpCAM expression between $\mathrm{PCa}$ and normal prostatic tissues was determined using the t-test. The relationship between EpCAM expression and the clinical features was analyzed using the univariate logistic regression model. Survival curve were analyzed by Kaplan-Meier method and log-rank test. The univariate and multivariate Cox proportional-hazards regression analyses were used to determine the influence of EpCAM expression on survival. The patients were divided into high and low expression groups based on the median value of EpCAM expression. TCGA data were conducted using $\mathrm{R}$ version 3.5.1 (R Core Team, 2018).

\section{Results}

Characteristics of the eligible studies from meta-analysis

Figure 1 shows the detailed procedure of the selecting the publications. Based on the selection criteria, seven studies were finally selected; in these studies, immunohistochemistry (IHC) was performed to determine EpCAM expression in patients with PCa [22, 23, 27, 33-36], (PCa tissue samples, $n=671$; normal prostatic tissue samples, $n=30$; benign prostatic tissue samples, $n=170$ ). Four studies involving 536 patients with $\mathrm{PCa}$ reported data related to
EpCAM expression and clinicopathological characteristics $[27,33,34,36]$. One study reported the prognostic information using multivariate Cox proportional-hazards regression analysis in patients with $\mathrm{PCa}$. The basic characteristics of the included publications are listed in Table 1.

\section{Association between EpCAM expression and PCa risk from meta-analysis}

175 PCa tissue samples were compared with 170 benign prostatic tissue samples and $33 \mathrm{PCa}$ tissue samples were compared with 30 normal prostatic tissue samples (Table 2). The results showed that EpCAM expression in PCa was significantly higher than in benign and normal tissue samples $\quad(\mathrm{OR}=77.93,95 \% \mathrm{CI}=4.90-1238.45, \quad P=0.002$; $\mathrm{OR}=161.61,95 \% \mathrm{CI}=17.65-1479.55, P<0.001$; respectively; Fig. 2).

Significant heterogeneity was detected between $\mathrm{PCa}$ and benign prostatic tissue samples $(P=0.043<0.1)$. To evaluate the change in pooled $\mathrm{OR}$ and heterogeneity based on the omission of an individual study, a sensitivity analysis was performed (PCa versus benign controls). When the study of Ni 2013 et al. [22] was removed, the re-calculated OR was 237.96 (95\% CI $=32.59-1737.73$, $P<0.001)$, resulting in a significantly decreased heterogeneity $(P=0.242)$.

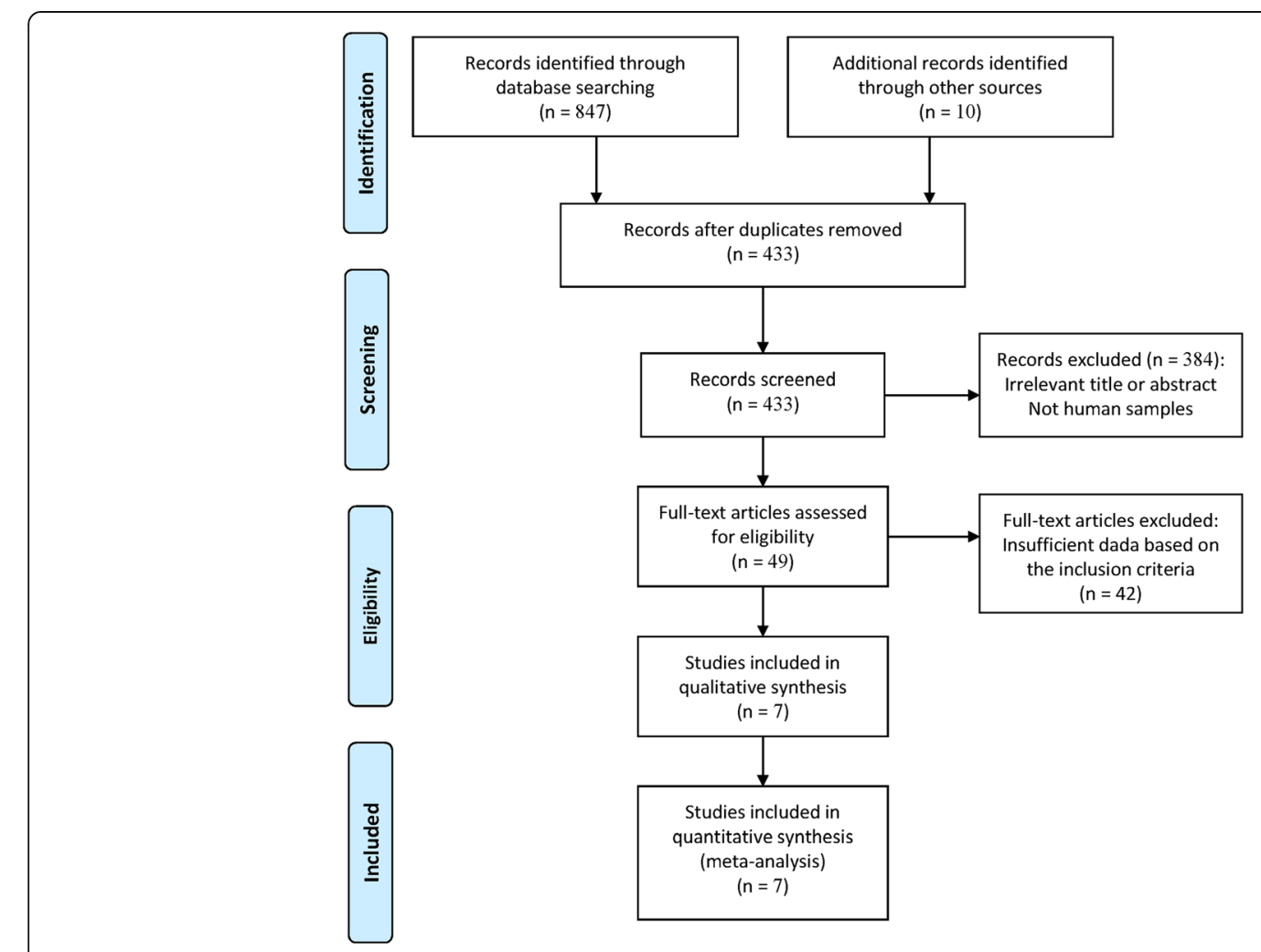

Fig. 1 Flow diagram of the study selection 


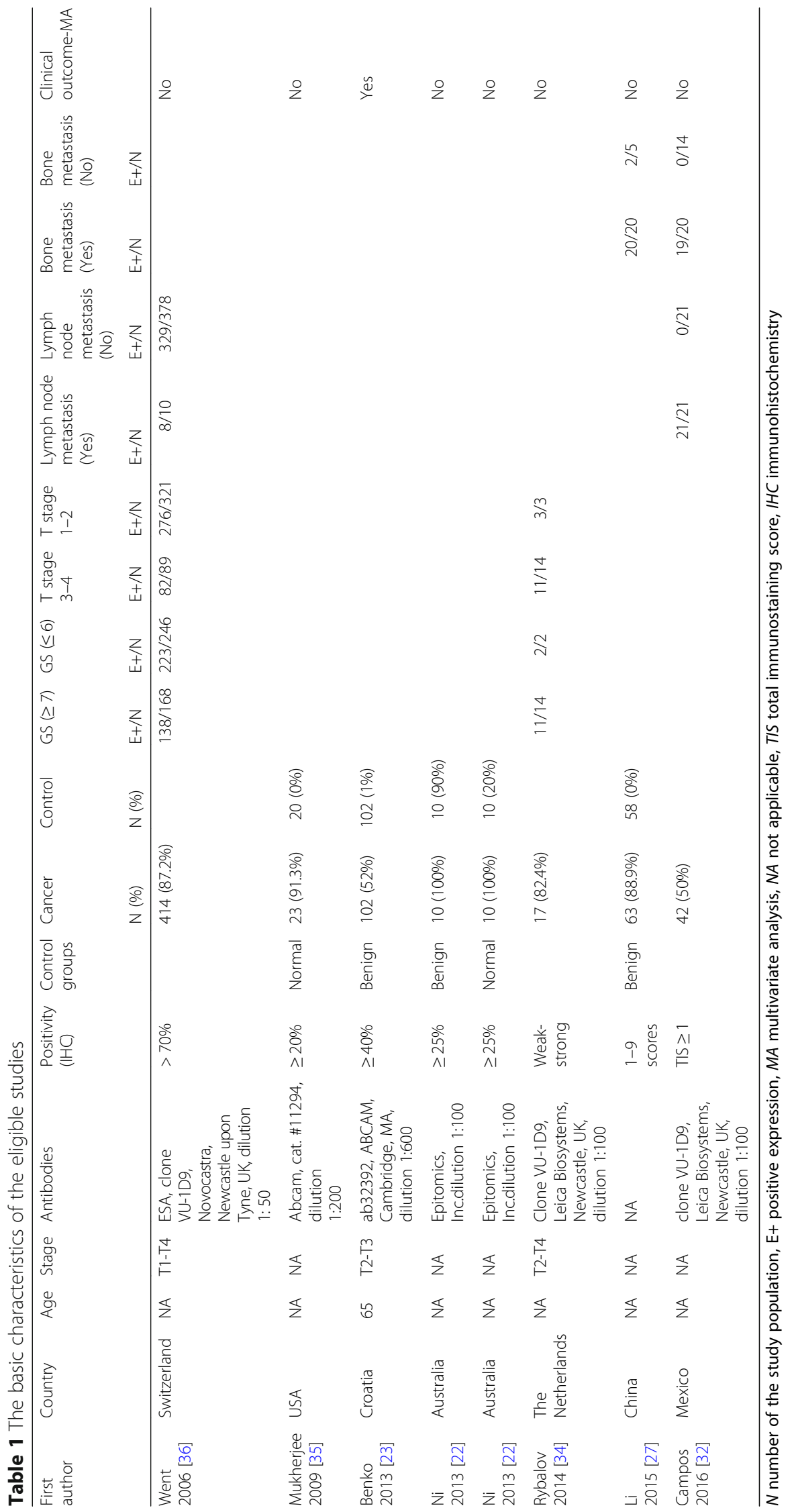


Table 2 Summary of the pooled results from meta-analysis

\begin{tabular}{|c|c|c|c|c|c|c|}
\hline Group & Studies & Cancer samples & Nonmalignant samples & OR with $95 \% \mathrm{Cl}$ & P & SE \\
\hline Cancer vs. Benign & 3 & 175 & 170 & $77.93(4.90-1238.45)$ & 0.002 & 1.411 \\
\hline Cancer vs. Normal & 2 & 33 & 30 & $161.61(17.65-1479.55)$ & $<0.001$ & 1.13 \\
\hline \multicolumn{7}{|l|}{ Clinicopathological characteristics } \\
\hline Gleason score ( $\geq 7$ vs. $\leq 6)$ & 2 & 430 & & $0.48(0.27-0.85)$ & 0.012 & 0.293 \\
\hline T stage (T3-4 vs. T1-2) & 2 & 427 & & $1.75(0.78-3.91)$ & 0.175 & 0.411 \\
\hline Lymph node metastasis (Positive vs. Negative) & 2 & 430 & & $27.89(0.004-1.7 e+05)$ & 0.455 & 4.481 \\
\hline Bone metastasis (Positive vs. Negative) & 2 & 59 & & $145.80(14.58-1458.02)$ & $<0.001$ & 1.175 \\
\hline
\end{tabular}

OR odds ratio, 95\% Cl 95\% confidence interval, SE standard error

\section{Association between EpCAM expression and} clinicopathological characteristics from meta-analysis

Data from two studies with 427 patients with PCa showed that EpCAM overexpression was not linked to $\mathrm{pT}$ stage $(P=0.175)$. Data from two studies with 430 PCa cases demonstrated no relationship between EpCAM overexpression and lymph node metastasis $(P=0.455$; Fig. 3$)$.

Data from two studies with 430 PCa cases demonstrated that EpCAM overexpression was associated with Gleason score $(\geq 7$ vs. $\leq 6$ scores: $\mathrm{OR}=0.48,95 \% \mathrm{CI}=0.27-0.85$, $P=0.012$; Fig. 3). Data from two studies with 59 patients with $\mathrm{PCa}$ showed that EpCAM overexpression was closely correlated to bone metastasis (OR $=145.80$, 95\% CI = 14.58-1458.02, $P<0.001$; Fig. 3). Moreover, no heterogeneity was measured between EpCAM overexpression and the Gleason score and bone metastasis of PCa $(P>0.1)$.

\section{Association between EpCAM expression and PCa prognosis from meta-analysis}

A study reported that EpCAM overexpression was significantly correlated with 5-year worse biochemical recurrence free-survival using multivariate Cox proportional-hazards regression analysis $(\mathrm{HR}=6.79,95 \% \mathrm{CI}=2.38-19.45)$ in 102 patients with $\mathrm{PCa}$ [23].

\section{TCGA}

Four hundred ninety-five PCa cases with the available clinical information and 52 normal prostatic tissue samples were identified from TCGA. EpCAM expression level was notably higher in $\mathrm{PCa}$ than in normal prostatic tissues $(P<0.001$; data not shown).

Univariate analysis using logistic regression showed that EpCAM overexpression was not significantly associated with the clinicopathological characteristics of $\mathrm{PCa}$

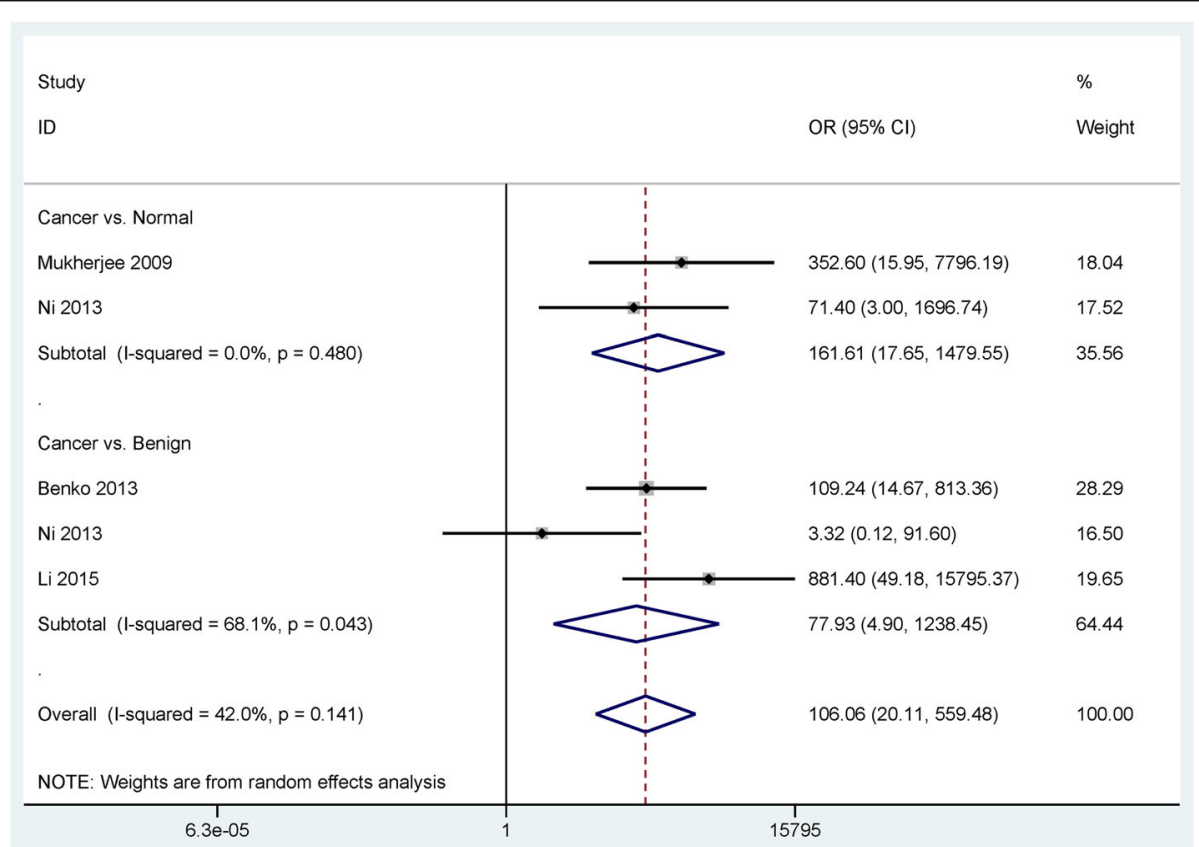

Fig. 2 Forest plot of the association between EpCAM immunoexpression and prostate cancer 


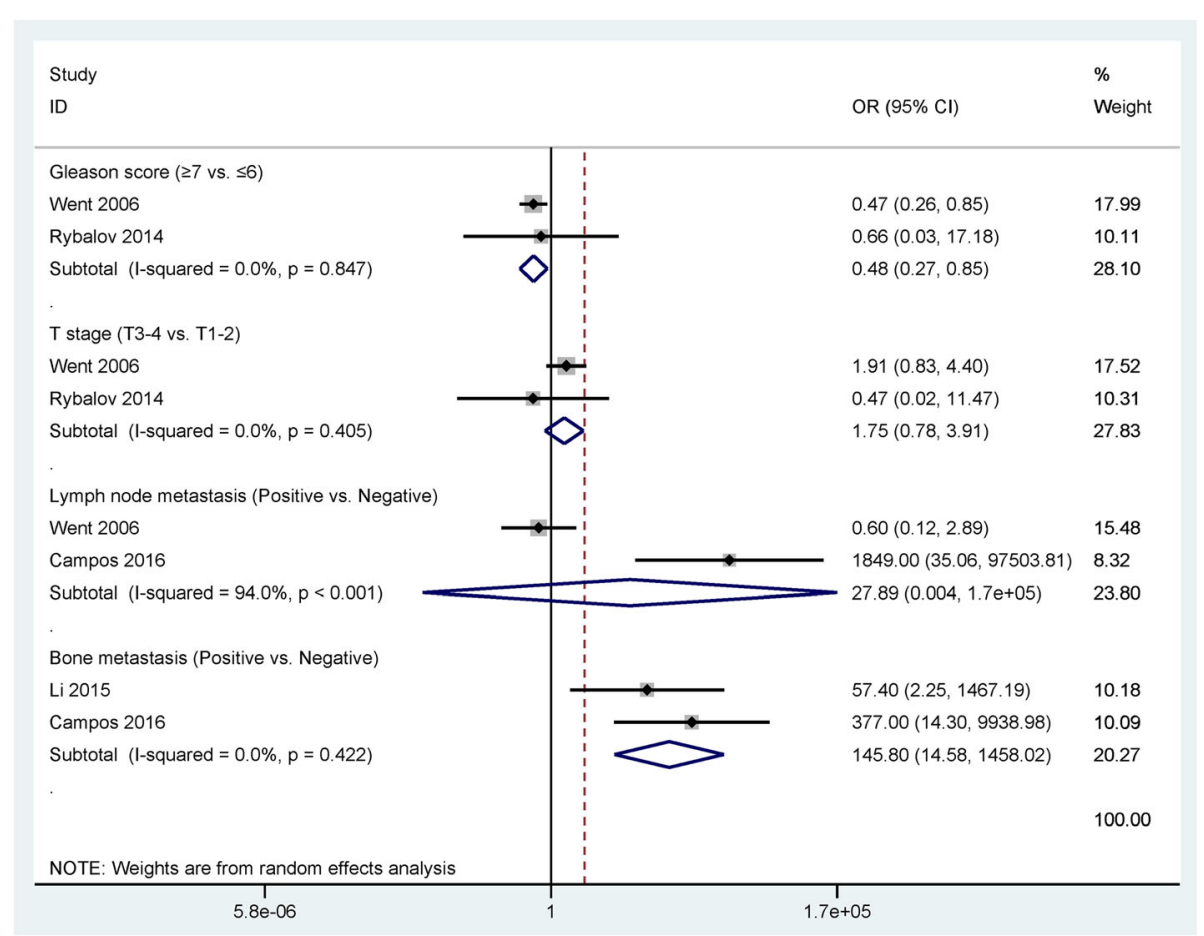

Fig. 3 Forest plot of the association of EpCAM immunoexpression with the clinicopathological features of patients with prostate cancer

(Table 3$)$, including age $(P=0.065)$, pT stage (T3-4 vs. T1-2: $P=0.78$ ), lymph node metastasis (positive vs. negative: $P=0.889$ ), number of lymph node ( $>10 \mathrm{vs.} \leq 10$ : $P=0.39$ ), prostate-specific antigen (PSA) value ( $\geq 10$ vs. $<$ 10: $P=0.288$ ), Gleason score ( $\geq 7$ vs. $\leq 6: P=0.561$ ), and biochemical recurrence (yes vs. no: $P=0.408$ ).

To further evaluate the prognostic value of EpCAM overexpression in patients with $\mathrm{PCa}$, Kaplan-Meier survival analysis was performed. This revealed that EpCAM overexpression was not correlated with the prognosis of overall survival $(P=0.25$; data not shown).

Table 3 Association of EpCAM overexpression with the clinicopathological characteristics from The Cancer Genome Atlas (TCGA)

\begin{tabular}{|c|c|c|c|}
\hline Factors & Total (N) & OR with $95 \% \mathrm{Cl}$ & $P$ \\
\hline Age $(\geq 61$ vs. <61) & 495 & $0.72(0.5-1.02)$ & 0.065 \\
\hline T stage (T3-4 vs. T1-2) & 488 & $0.95(0.66-1.37)$ & 0.78 \\
\hline $\begin{array}{l}\text { Lymph node metastasis } \\
\text { (Positive vs. Negative) }\end{array}$ & 422 & $1.04(0.63-1.69)$ & 0.889 \\
\hline $\begin{array}{l}\text { Number of lymph node } \\
(>10 \text { vs. } \leq 10)\end{array}$ & 420 & $1.18(0.81-1.74)$ & 0.39 \\
\hline $\begin{array}{l}\text { Biochemical recurrence } \\
\text { (Yes vs. No) }\end{array}$ & 427 & $0.79(0.45-1.38)$ & 0.408 \\
\hline Gleason score $(\geq 7$ vs. $\leq 6)$ & 483 & $1.19(0.66-2.14)$ & 0.561 \\
\hline PSA value $(\geq 10$ vs. $<10)$ & 438 & $1.75(0.62-4.89)$ & 0.288 \\
\hline
\end{tabular}

$N$ number of the study population, PSA prostate-specific antigen, OR odds ratio, $95 \% \mathrm{Cl}$ 95\% confidence interval

\section{Discussion}

CSCs, a special subpopulation of tumor cells, have the ability to form the bulk of the tumor and contribute to tumor progression and relapse [9]. Therefore, eradication of CSCs is considered as a crucial challenge for a successful cancer therapy [37]. EpCAM is identified as a CSC marker that is closely linked to tumor progression [38]. EpCAM plays an important role in the cell-cell adhesion, cell signaling, migration, proliferation, and differentiation [39]. EpCAM also plays an important role in mediating migration of immune cells [38]. EpCAM in PCa is associated with tumor progression and metastasis and therapeutic resistance via the PI3K/Akt/mTOR signaling pathway [22]. EpCAM is frequently overexpressed across a wide range of human cancer, including pancreatic adenocarcinoma, breast cancer, ovarian cancer, and head and neck squamous cell cancer [40]. EpCAM overexpression is correlated with worse prognosis in some types of cancer $[19,41,42]$. Some studies reported that EpCAM is highly expressed in patients with $\mathrm{PCa}[22,27,33,34]$. However, the function of EpCAM and its clinical effect in PCa remains largely unclear. In this study, we analyzed the clinicopathological significance of EpCAM expression and its role in the prognosis of PCa.

The pooled data demonstrated that EpCAM expression was significantly higher in PCa than in benign and normal prostatic tissue samples, suggesting that EPCAM overexpression may be associated with the development 
of PCa. Our results were consistent with the previous studies that EpCAM was expressed more frequently in $\mathrm{PCa}$ than in benign $[23,27]$ and normal prostatic tissue samples [22, 35]. Moreover, TCGA data also showed that EpCAM expression was notably increased in $\mathrm{PCa}$ than in normal tissue samples.

We further analyzed whether EpCAM overexpression was correlated with the clinicopathological characteristics of patients with PCa. EpCAM overexpression was not linked to $\mathrm{pT}$ stage and lymph node metastasis, but was associated with Gleason score and bone metastasis. TCGA data revealed that EpCAM overexpression was not closely correlated with the clinicopathological characteristics in 495 patients with $\mathrm{PCa}$, including age, pT stage, lymph node metastasis, number of lymph node, prostate-specific antigen (PSA) level, Gleason score, and biochemical recurrence. The pooled analyses suggested that EpCAM overexpression may predict bone metastasis. However, as the sample size was small, the relationship between EpCAM overexpression and bone metastasis requires further investigation.

EpCAM overexpression was reported to be closely associated with 5-year worse biochemical recurrence freesurvival using multivariate Cox proportional-hazards regression analysis in 102 patients with $\mathrm{PCa}$ [23]; this suggests that EpCAM overexpression may be a potential prognostic biomarker for predicting poor biochemical recurrence free-survival. However, no association was found between EpCAM overexpression and overall survival of patients with $\mathrm{PCa}$.

Our result showed no correlation between EpCAM overexpression and lymph node metastasis among two studies with 430 patients with PCa, which was consistent with the study of Went 2006 et al. [36] among a large cohort (388 patients). Heterogeneity was found in the correlation of $\mathrm{PCa}$ and benign prostatic tissue samples $(P=0.043)$. When we removed the study by Ni 2013 et al. [22], the re-calculated OR remained significant with no obvious evidence of heterogeneity. The analysis suggested that our results were reliable. The reasons of the heterogeneity were not very clear; the inappropriate conditions of IHC methods may cause the bias.

There were some limitations in the current study. First, as none of the present meta-analyses included $\geq 10$ studies, we could not examine and rule out the risk of the potential publication bias. Although we systematically searched PubMed, EMBASE, Cochrane Library, EBSCO, Wanfang, and CNKI databases to minimize the potential bias, the eligible studies were restricted to papers published in English or Chinese. Moreover, studies published in language other than English and Chinese, unpublished papers, or conference abstracts were excluded based on incomplete information. Furthermore, studies with negative results are less likely to be published than papers with positive results. These reasons may cause potential bias. Second, the main ethnic population in this meta-analysis was European; other ethnic groups, such as Asians and Africans, were underrepresented. Third, the studies regarding the associations of EpCAM overexpression with bone metastasis and biochemical recurrence free-survival were relatively small. Further studies with large sample size are needed to further confirm these results. Finally, the cut-off values of EpCAM expression were different among some studies, which showed inconsistent definitions for "negative" and "positive," leading to the potential heterogeneity. Therefore, the results need to be interpreted with caution. EpCAM expression should be defined as positive or negative based on a uniform standard in the future.

\section{Conclusions}

In conclusion, the current meta-analysis suggested that EpCAM expression was significantly high in PCa than in benign and normal prostatic tissue samples. EpCAM overexpression was not associated with age, $\mathrm{pT}$ stage, lymph node metastasis, number of lymph node, prostate-specific antigen (PSA) level, biochemical recurrence, and overall survival. EpCAM overexpression may be related to the development of bone metastasis and worse biochemical recurrence free-survival of $\mathrm{PCa}$. Our results should be interpreted with caution owing to the small sample size. Additional large-scale prospective studies are required to further validate our findings.

\section{Abbreviations}

Cls: Confidence intervals; CSCs: Cancer stem cells; EpCAM: Epithelial cell adhesion molecule; ESA: Epithelial-specific antigen; HRs: hazard ratios; IHC: Immunohistochemistry; ORs: The odds ratios; PCa: Prostate cancer; PSA: Prostate-specific antigen; TCGA: The Cancer Genome Atlas

\section{Acknowledgments}

None.

\section{Authors' contributions}

Study conception and design: WYT, HY, WQ. Manuscript writing and editing: HY and WQ. Data collection: HY, WQ, GJL, ZYR. Data management/analysis: HY, WQ, GJL, ZYR, WYT. All authors read and approved the final manuscript.

\section{Funding}

The study was supported by the program of National Natural Science Foundation of China (NSFC) (81671574). The funding agencies and donors had no role in any aspect of this study.

\section{Availability of data and materials}

The datasets used and/or analyzed during the current study are available from the corresponding author on reasonable request.

Ethics approval and consent to participate

Not applicable.

Consent for publication

Not applicable.

Competing interests

The authors declare that they have no conflict of interest. 


\section{Author details}

'Department of Pathology, China-Japan Union Hospital, Jilin University, Changchun, Jilin, China. ${ }^{2}$ Department of Urology, The First Hospital of Jilin University, No. 71 Xinmin Street, Changchun 130021, Jilin, China.

\section{Received: 16 April 2019 Accepted: 9 July 2019}

\section{Published online: 19 July 2019}

\section{References}

1. Bray F, Ferlay J, Soerjomataram I, Siegel RL, Torre LA, Jemal A. Global cancer statistics 2018: GLOBOCAN estimates of incidence and mortality worldwide for 36 cancers in 185 countries. CA Cancer J Clin. 2018;68(6):394-424.

2. Hussein AA, Punnen S, Zhao S, Cowan JE, Leapman M, Tran TC, Washington SL, Truesdale MD, Carroll PR, Cooperberg MR. Current use of imaging after primary treatment of prostate Cancer. J Urol. 2015;194(1):98-104.

3. Fossa SD, Nilssen Y, Kvale R, Hernes E, Axcrona K, Moller B. Treatment and 5-year survival in patients with nonmetastatic prostate cancer: the Norwegian experience. Urology. 2014;83(1):146-52.

4. Norum J, Nieder C. Treatments for metastatic prostate Cancer (mPC): a review of costing evidence. Pharmacoeconomics. 2017;35(12):1223-36.

5. Crawford ED, Petrylak D, Sartor O. Navigating the evolving therapeutic landscape in advanced prostate cancer. Urol Oncol. 2017:35S:S1-S13.

6. Han S, Huang T, Li W, Wang X, Wu X, Liu S, Yang W, Shi Q, Li H, Hou F. Prognostic value of CD44 and its isoforms in advanced Cancer: a systematic meta-analysis with trial sequential analysis. Front Oncol. 2019;9:39.

7. Steinbichler TB, Dudas J, Skvortsov S, Ganswindt U, Riechelmann H, Skvortsova II. Therapy resistance mediated by cancer stem cells. Semin Cancer Biol. 2018:53:156-67.

8. Han S, Huang T, Wu X, Wang X, Liu S, Yang W, Shi Q, Li H, Hou F. Prognostic value of CD133 and SOX2 in advanced Cancer. J Oncol. 2019:2019:3905817.

9. Koren E, Fuchs $Y$. The bad seed: Cancer stem cells in tumor development and resistance. Drug Resist Updat. 2016;28:1-12.

10. Mueller MT, Hermann PC, Heeschen C. Cancer stem cells as new therapeutic target to prevent tumour progression and metastasis. Front Biosci (Elite Ed). 2010;2:602-13.

11. Balzar M, Winter MJ, de Boer CJ, Litvinov SV. The biology of the 17-1A antigen (ep-CAM). J Mol Med (Berl). 1999;77(10):699-712.

12. Litvinov SV, Bakker HA, Gourevitch MM, Velders MP, Warnaar SO. Evidence for a role of the epithelial glycoprotein 40 (ep-CAM) in epithelial cell-cell adhesion. Cell Adhes Commun. 1994;2(5):417-28.

13. Ni J, Cozzi PJ, Hao JL, Beretov J, Chang L, Duan W, Shigdar S, Delprado WJ, Graham PH, Bucci J, et al. CD44 variant 6 is associated with prostate cancer metastasis and chemo-/radioresistance. Prostate. 2014;74(6):602-17.

14. Chen X, Rycaj K, Liu X, Tang DG. New insights into prostate cancer stem cells. Cell Cycle. 2013;12(4):579-86.

15. Salnikov AV, Groth A, Apel A, Kallifatidis G, Beckermann BM, Khamidjanov A, Ryschich E, Buchler MW, Herr I, Moldenhauer G. Targeting of cancer stem cell marker EpCAM by bispecific antibody EpCAMxCD3 inhibits pancreatic carcinoma. J Cell Mol Med. 2009;13(9B):4023-33.

16. Han S, Zong S, Shi Q, Li H, Liu S, Yang W, Li W, Hou F. Is ep-CAM expression a diagnostic and prognostic biomarker for colorectal Cancer? A systematic meta-analysis. EBioMedicine. 2017;20:61-9.

17. Dai M, Yuan F, Fu C, Shen G, Hu S, Shen G. Relationship between epithelial cell adhesion molecule (EpCAM) overexpression and gastric cancer patients: a systematic review and meta-analysis. PLoS One. 2017;12(4):e0175357.

18. Cimino A, Halushka M, Illei P, Wu X, Sukumar S, Argani P. Epithelial cell adhesion molecule (EPCAM) is overexpressed in breast cancer metastases. Breast Cancer Res Treat. 2010;123(3):701-8.

19. Schmidt M, Hasenclever D, Schaeffer M, Boehm D, Cotarelo C, Steiner E, Lebrecht A, Siggelkow W, Weikel W, Schiffer-Petry I, et al. Prognostic effect of epithelial cell adhesion molecule overexpression in untreated node-negative breast cancer. Clin Cancer Res. 2008;14(18):5849-55.

20. Shim HS, Yoon BS, Cho NH. Prognostic significance of paired epithelial cell adhesion molecule and E-cadherin in ovarian serous carcinoma. Hum Pathol. 2009;40(5):693-8

21. Zhou L, Zhu Y. The EpCAM overexpression is associated with clinicopathological significance and prognosis in hepatocellular carcinoma patients: a systematic review and meta-analysis. Int J Surg. 2018;56:274-80.

22. Ni J, Cozzi P, Hao J, Beretov J, Chang L, Duan W, Shigdar S, Delprado W, Graham P, Bucci J, et al. Epithelial cell adhesion molecule (EpCAM) is associated with prostate cancer metastasis and chemo/radioresistance via the PI3K/Akt/mTOR signaling pathway. Int J Biochem Cell Biol. 2013;45(12):2736-48

23. Benko G, Spajic B, Kruslin B, Tomas D. Impact of the EpCAM expression on biochemical recurrence-free survival in clinically localized prostate cancer. Urol Oncol. 2013;31(4):468-74.

24. Elschot M, Selnaes KM, Sandsmark E, Kruger-Stokke B, Storkersen O, Giskeodegard GF, Tessem MB, Moestue SA, Bertilsson H, Bathen TF. Combined (18) F-Fluciclovine PET/MRI shows potential for detection and characterization of high-risk prostate Cancer. J Nucl Med. 2018;59(5):762-8.

25. Merseburger AS, Kramer MW, Hennenlotter J, Simon P, Knapp J, Hartmann JT, Stenzl A, Serth J, Kuczyk MA. Involvement of decreased Galectin-3 expression in the pathogenesis and progression of prostate cancer. Prostate. 2008;68(1):72-7

26. Henshall SM, Afar DE, Rasiah KK, Horvath LG, Gish K, Caras I, Ramakrishnan V, Wong M, Jeffry U, Kench JG, et al. Expression of the zinc transporter ZnT4 is decreased in the progression from early prostate disease to invasive prostate cancer. Oncogene. 2003;22(38):6005-12.

27. Li ZL, Jin RS. Expression of the epithelial cell adhesion molecule in prostate cancer and its clinical significance. Zhonghua Nan Ke Xue. 2015;21(4):320-4.

28. Liberati A, Altman DG, Tetzlaff J, Mulrow C, Gotzsche PC, loannidis JP, Clarke M, Devereaux PJ, Kleijnen J, Moher D. The PRISMA statement for reporting systematic reviews and meta-analyses of studies that evaluate health care interventions: explanation and elaboration. PLoS Med. 2009:6(7):e1000100.

29. Zintzaras E, loannidis JP. HEGESMA: genome search meta-analysis and heterogeneity testing. Bioinformatics. 2005;21(18):3672-3.

30. Han S, Huang T, Wu X, Wang X, Li W, Liu S, Yang W, Shi Q, Li H, Shi K, et al. Prognostic value of ALDH1 and nestin in advanced cancer: a systematic meta-analysis with trial sequential analysis. Ther Adv Med Oncol. 2019;11:1758835919830831.

31. Higgins JP, Thompson SG, Deeks JJ, Altman DG. Measuring inconsistency in meta-analyses. BMJ. 2003;327(7414):557-60.

32. Egger M, Davey Smith G, Schneider M, Minder C. Bias in meta-analysis detected by a simple, graphical test. BMJ. 1997;315(7109):629-34.

33. Campos AK, Hoving HD, Rosati S, van Leenders GJ, de Jong IJ. EpCAM Expression in Lymph Node and Bone Metastases of Prostate Carcinoma: A Pilot Study. Int J Mol Sci. 2016:17(10):9.

34. Rybalov M, Ananias HJ, Hoving HD, van der Poel HG, Rosati S, de Jong IJ. PSMA, EpCAM, VEGF and GRPR as imaging targets in locally recurrent prostate cancer after radiotherapy. Int J Mol Sci. 2014;15(4):6046-61.

35. Mukherjee S, Richardson AM, Rodriguez-Canales J, Ylaya K, Erickson HS, Player A, Kawasaki ES, Pinto PA, Choyke PL, Merino MJ, et al. Identification of EpCAM as a molecular target of prostate cancer stroma. Am J Pathol. 2009:175(6):2277-87.

36. Went $P$, Vasei M, Bubendorf L, Terracciano L, Tornillo L, Riede $U$, Kononen J, Simon R, Sauter G, Baeuerle PA. Frequent high-level expression of the immunotherapeutic target ep-CAM in colon, stomach, prostate and lung cancers. Br J Cancer. 2006:94(1):128-35.

37. Kuhlmann JD, Hein L, Kurth I, Wimberger P, Dubrovska A. Targeting Cancer stem cells: promises and challenges. Anti Cancer Agents Med Chem. 2016;16(1):38-58.

38. Martowicz A, Seeber A, Untergasser G. The role of EpCAM in physiology and pathology of the epithelium. Histol Histopathol. 2016;31(4):349-55.

39. Ni J, Cozzi PJ, Duan W, Shigdar S, Graham PH, John KH, Li Y. Role of the EpCAM (CD326) in prostate cancer metastasis and progression. Cancer Metastasis Rev. 2012;31(3-4):779-91.

40. Moldenhauer G, Salnikov AV, Luttgau S, Herr I, Anderl J, Faulstich H. Therapeutic potential of amanitin-conjugated anti-epithelial cell adhesion molecule monoclonal antibody against pancreatic carcinoma. J Natl Cancer Inst. 2012;104(8):622-34.

41. Matsuda T, Takeuchi H, Matsuda S, Hiraiwa K, Miyasho T, Okamoto M, Kawasako K, Nakamura R, Takahashi T, Wada N, et al. EpCAM, a potential therapeutic target for esophageal squamous cell carcinoma. Ann Surg Oncol. 2014;21(Suppl 3):S356-64.

42. Prince S, Zeidman A, Dekel Y, Ram E, Koren R. Expression of epithelial cell adhesion molecule in gallbladder carcinoma and its correlation with clinicopathologic variables. Am J Clin Pathol. 2008;129(3):424-9.

\section{Publisher's Note}

Springer Nature remains neutral with regard to jurisdictional claims in published maps and institutional affiliations. 